\title{
Herbal medicinal products and phytotherapy-a thematic issue
}

\author{
Brigitte Kopp
}

Published online: 29 March 2017

(C) Springer-Verlag Wien 2017

Until the early 19th century, medicinal plants were the most important and irreplaceable sources of therapeutics. With the development of plant chemistry since the 18th century, culminating in the isolation of morphine 1805 by the apothecary F.W.A. Sertürner, attention finally turned to single substances, their derivatives and synthetic molecules, which could be quickly produced on an industrial level and were thought to be better controllable in terms of dosage, efficacy and side effects. In the progress of this development, many medicinal plants fell into oblivion, were not further investigated or not even studied.

During the last decades, scientific interest has turned again to medicinal plants, as research in tradition, compounds and composition might reveal new opportunities for future challenges and essential medicinal needs, not only under the aspect of "soft therapeutics", but also as a source of clearly effective resources for the treatment of diseases, including serious diseases, with presently unsatisfying therapeutic solutions.

Today, we have new methods and much more opportunity to explore the composition of plants under the aspect of their efficacy more deeply than ever before. This knowledge can now be linked to pharmacological and clinical studies, to allow a much better understanding of herbal medicines, their pharmacological (incl. toxicological) effects and their effectiveness.

In contrast to most synthetic chemical ingredients and isolated pure substances of biogenic origin, the active ingredient of herbal medicines is a multicomponent mixture with high quality demands. The ad-

Univ.-Prof.i.R. Mag. Dr.Dr.h.c. B. Kopp $(\bowtie)$

Department of Pharmcognosy, University of Vienna,

Althanstrasse 14, 1090 Vienna, Austria

brigitte.kopp@univie.ac.at vantage of this multicompound pleiotropic preparation (herbal medicinal product) is often precisely the fact that a number of substances with various molecular mechanisms contribute to the desired overall effectiveness. Evidence of efficacy in clinical trials has shown that herbal medicinal products have the right to occupy a firm place in modern drug therapy.

Phytotherapeuticals are generally regarded as having a relatively low risk. Despite the fact that side effects and interactions cannot be excluded, a special feature of herbal medicines is that they usually have a lower rate of side effects than synthetic drugs.

Many medicinal plants have been used as teas, powdered medicines, or alcoholic extracts (tinctures, drops) for decades or even centuries. Thanks to intensive research and modern technology, today, extracts can be concentrated and marketed as capsules or tablets, which contain the active ingredients of the plant.

Today, the emotional perception of herbal medicinal products oscillates between enthusiastic acceptance and radical rejection. Unfortunately, supporters and opponents argue mostly on a fundamental level and hardly differentiate. Therefore, the intention is to present information in an objective, independent and fair manner, so that emotional arguments are replaced by correct factual arguments. Supporters and opponents also talk about some very different things, such as herbal medicinal products and dietary supplements (functional food, etc.), which are increasingly present on the market. However, dietary supplements are legally classified as foods and therefore only suitable for the maintenance of good health. Although dietary supplements may also be of plant origin, they are not medicines because they neither have to demonstrate pharmaceutical quality, pharmacological effects or efficacy, nor safety (adverse effects, toxicity, etc.). 
In appearance, dietary supplements are very similar to medicines. This causes great danger for the patient: The dietary supplements are used by the patient in the form of self-medication, but critical evaluation (by the doctor, pharmacist, leaflet) is missing. This direction currently represents a massive threat for phytopharmaceuticals.

How can a patient recognize weather a pill is a medicine or not? Each drug is allocated a drug approval number according to the pharmaceutical legislation. Dietary supplements do not have this authorization number. Efficacy and safety can be expected only from medicines, as they must be tested before their approval. Given the confusing offers of the Internet and drugstores, there is an urgent need for consultation strategies and targeted information regarding the characteristics and marketing of dietary supplements and medicines.

It is important that doctors and pharmacists receive adequate and scientifically sound information about phytopharmaceuticals during their training, to educate patients regarding the quality, safety and efficacy of the herbal medicines. In addition, the patient should discuss self-medication with herbal drugs with the doctor and/or pharmacist, to prevent possible secondary effects or interactions, and to coordinate their use with his/her overall individual therapy.

The growing demand for herbal medicinal products is accompanied by a high demand for information, which can only be met by intensive and systematic research.
The results contained in this special issue show the importance of research activity for the increase in evidence regarding quality, safety and proof of effectiveness of herbal medicinal products.

In addition to a review article regarding " 60 years of Cimicifuga racemosa medicinal products-Clinical research milestones, current study findings and development", another contribution addresses the mechanisms on spasmolytic and anti-inflammatory effects of a herbal medicinal product consisting of myrrh, chamomile flower and coffee charcoal.

In the following issue, the use of herbal medicinal products for lower urinary tract symptoms (LUTS), motility modulation beyond metoclopramide and domperidone, and pharmacological and clinical evidence for phytotherapy in functional gastrointestinal disorders will be discussed. In addition, the problem of ethanol in herbal medicinal products for children will be presented, as well as a clinical trial showing lasting function of a new moisturizing cream against vaginal dryness.

We hope that this special issue will increase the understanding of the value and possible use of herbal medicinal products, and we thank the authors for their contributions, which support the scientific progress of phytotherapy.

Conflict of interest B. Kopp declares that she has no competing interests. 